\title{
LA PRIMA AMBASCIATA ETIOPICA IN OCCIDENTE (1315 CA.) SVELATA DA UN CRONISTA MILANESE
}

\author{
Nota del s.c. PAOLO CHIESA (*)
}

(Adunanza del 23 maggio 2019)

SunTO. - Un passo dell'inedita Cronica universalis del domenicano milanese Galvano Fiamma (m. 1345 ca.) contiene una notizia relativa alla spedizione compiuta nel 1291 da due navi genovesi, che si proponeva di raggiungere l'India per la via dell'Atlantico. Tale spedizione era nota finora soltanto dagli Annales Ianuenses, che ne attribuivano il comando ai fratelli Vivaldi; nel racconto di Galvano compare un nome diverso, quello di Uberto di Savignone (un castello non lontano da Genova). La fonte di Galvano è il perduto libellus scritto da un prete genovese, Giovanni da Carignano, che dice di aver tratto le sue informazioni da un'ambasciata giunta dall'Etiopia intorno al 1315; gli ambasciatori raccontarono che i Genovesi erano giunti nella loro terra, ma avevano rinunciato a tornare in patria per le difficoltà del viaggio. Il nuovo testo anticipa di un secolo i primi contatti conosciuti fra Etiopia e Europa occidentale nel medioevo.

$* * *$

ABSTRACT. - A passage from the unpublished Cronica universalis by the Milanese Dominican Galvano Fiamma (d. c. 1345) contains news about the expedition carried out in 1291 by two Genoese ships, which was intended to reach India by the Atlantic route. Until now, this expedition was known only from the Annales Ianuenses; according this text, the commanders of the expedition were the Vivaldi brothers, although in Galvano's account the name of the admiral is Ubertus of Savignone. Galvano's source is a lost libellus written by a Genoese priest, Giovanni da Carignano, who said he got information about the sailors' fate from an embassy that came from Ethiopia around 1315: the ambassadors told that the Genoese had arrived in their land, but had given up returning home because of the difficulties of the journey. Galvano's new witness anticipates by a century the first known contacts between Ethiopia and Western Europe in the middle ages.

(") Dipartimento di Studi Letterari Filologici e Linguistici, Università degli Studi di Milano, Italy. E-mail: paolo.chiesa@unimi.it 
In una delle ultime pagine degli Annales della città di Genova, scritti da Iacopo Doria, si legge questa breve notizia su un fatto avvenuto nel 1291:

Eodem quippe anno Thedisius Aurie, Ugolinus de Vivaldus et eius frater cum quibusdam aliis civibus Ianue, ceperunt facere quoddam viagium quod aliquis usque tunc facere minime attemptavit. Nam armaverunt optime duas galeas, et victualibus, aqua et aliis necessariis eis impositis, miserunt eas de mense Madii de versus strictum Septe, ut per mare occeanum irent ad partes Indie mercimonia utilia inde deferentes. In quibus iverunt dicti duo fratres de Vivaldo personaliter, et duo fratres Minores; quod quidem mirabile fuit non solum videntibus sed etiam audientibus. Et postquam locum qui dicitur Gozora transierunt, aliqua certa nova non habuimus de eis. Dominus autem eos custodiat, et sanos et incolumes reducat ad propria. ${ }^{1}$

In quell'anno, Tedisio Doria, Ugolino de' Vivaldi e suo fratello, con alcuni altri cittadini di Genova, intrapresero un viaggio che nessuno mai fino ad allora aveva tentato. Armarono di tutto punto due galee, vi caricarono cibo, acqua e quanto altro serviva, e nel mese di Maggio le misero in mare dirette allo stretto di Ceuta: volevano raggiungere l'India navigando per l'Oceano, per riportare da lì prodotti utili. Su queste galee partirono i due fratelli Vivaldi in persona e due francescani. L'impresa parve un evento straordinario non solo a chi la vide, ma anche a chi ne sentì parlare. Passarono dalla località di Gozora; ma da lì in poi non abbiamo più saputo nulla di loro. Dio li protegga e li riporti a casa sani e salvi.

Da questa notizia veniamo a sapere che, duecento anni prima di Colombo, altri genovesi avevano cercato di raggiungere le Indie per la via dell'Atlantico; che avevano superato lo strictus Septe, cioè lo stretto di Gibilterra, e raggiunto Gozora, una località sulla costa atlantica del Marocco; che da lì se ne erano perse le tracce. Al di là dell'augurio di rito - Dominus eos custodiat -, il tono della frase finale di Iacopo pare piuttosto un congedo funebre che una dichiarazione di speranza; quando il cronista scriveva erano passati ormai tre anni, e non c'erano notizie. Per quanto sappiamo, a Genova i naviganti non ritornarono più.

La spedizione dei fratelli Vivaldi costituisce un episodio epico,

1 Annali genovesi di Caffaro e de' suoi continuatori, V: Dal 1280 al 1293, ed. C. Imperiale di Sant'Angelo, Roma, Tipografia del Senato, 1929, 124. 
anche se in gran parte misterioso, della storia della navigazione. ${ }^{2}$ Essa è stata talvolta chiamata in causa come possibile ispiratrice della vicenda dantesca di Ulisse, che, nel XXVI canto dell'Inferno, supera con la sua nave e i suoi compagni le colonne d'Ercole e da lì affronta l'Oceano procedendo verso meridione, in un'impossibile sfida all'ignoto che si conclude tragicamente. ${ }^{3}$ Non c'è in realtà nessuna prova che Dante conoscesse la vicenda, che è quasi ignorata dalle fonti dell'epoca: ${ }^{4}$ è pur vero però che, come è stato recentemente rilevato, ${ }^{5}$ scienziati e geografi di allora dibattevano molto sulla possibilità di vita e di viaggio nelle regioni del sud, a dispetto del caldo torrido, e su questi argomenti si scambiavano informazioni; e Dante si annoverava certo fra gli scienziati, mentre i Genovesi erano all'avanguardia fra i geografi. ${ }^{6}$ Il racconto di Iacopo, per altro, non chiarisce quale fosse la rotta che si proponevano di seguire i Vivaldi per raggiungere le Indie: forse quella meridionale, che volgeva poi a est lungo il grande fiume che - secondo alcune teorie - avrebbe attraversato l'Africa all'altezza dell'Equatore; ma forse quella occidentale che poi avrebbe percorso Colombo, perché, nonostante le opinioni vulgate, gli astronomi del medioevo avevano ben chiaro che la terra è una sfera, e ancor più chiaro dovevano averlo i marinai, avvezzi a fare i conti con la curvatura dell'orizzonte.

Che ne fu dei fratelli Vivaldi? L'epicità dell'impresa e l'oscurità

2 A. Magnaghi, Precursori di Colombo? Il tentativo di viaggio transoceanico dei genovesi fratelli Vivaldi nel 1291, Roma, Società Anonima Italiana Arti Grafiche, 1935 (Memorie della Reale Società Geografica Italiana 18); G. Moore, La spedizione dei fratelli Vivaldi e nuovi documenti d'archivio, Atti della Società Ligure di Storia Patria n.s. 12 (1972), 387-402 [trad. ingl. con aggiornamenti: The expedition of the Brothers Vivaldi: new archival evidence, in Spain, Portugal and the Atlantic frontier of medieval Europe, Farnham, Ashgate, 2013, 1-18]; F. Surdich, L'impresa dei fratelli Vivaldi, in Le Americhe annunciate. Viaggi ed esplorazioni liguri prima di Colombo, Reggio Emilia, Diabasis, 1991, pp. 59-71; A. Quartapelle, El «loco vuelo» de los hermanos Vivaldi en 1291, Revista de Historia Canaria 200 (apr. 2018), 227-249.

3 Cfr. B. Nardi, La tragedia d'Ulisse, Studi Danteschi 20 (1937), 5-15: 5-6; F. M. Rogers, The Vivaldi expedition, Annual Report of the Dante Society 73 (1955), 31-45.

4 Solo da Pietro d'Abano, Conciliator differentiarum philosophorum et medicorum, 67 (ed. F. Argilagnes, Venetiis 1496 [GW M31860, ISTC ip00435000], f. 98vb).

5 M. Tavoni, Dante e la scoperta del Paradiso terrestre in mezzo all'Oceano, Studi Danteschi 84 (2019), 1-14.

6 Soprattutto nel campo della cartografia: P. Revelli, Cristoforo Colombo e la scuola cartografica genovese, Genova, Stabilimenti Italiani Arti Grafiche, 1937. 
del suo esito stimola alla fantasia romanzesca. ${ }^{7}$ Una fonte recentemente scoperta ci fa conoscere una possibile sorte della spedizione: un esito probabilmente anch'esso fantastico, ma che era raccontato a Genova un quarto di secolo dopo la partenza della spedizione.

$$
* * *
$$

La nuova fonte è un testo milanese. Si tratta delle Cronica generalis sive universalis del domenicano Galvano Fiamma, un frate che apparteneva al convento di Sant'Eustorgio e che morì intorno al $1345 .{ }^{8}$ Egli sembra avere avuto un ruolo piuttosto rilevante come anello di collegamento fra il suo Ordine e i Visconti, che erano stati su posizioni contrarie nelle discordie che avevano opposto partito imperiale e partito papale nei primi decenni del Trecento; per i Visconti scrisse diverse opere storiografiche, quelle per le quali è ancora oggi noto. La sua produzione conta una quindicina di titoli," che fanno di lui il più prolifico scrittore del medioevo milanese; opere in verità di qualità letteraria scadente e di valore storico modesto. Galvano legge molto, ma usa male le sue letture: affastella disordinatamente le notizie che trova, dà credito all'incredibile, non sa discutere o dirimere i punti contraddittori, e in più scrive in un latino stentato. Oltre tutto è un velleitario: ambisce a essere il grande storico della città di Milano e della famiglia al potere, ma spesso non porta a termine le sue opere: le ricomincia da capo riciclando gli stessi materiali in una struttura diversa. Un cattivo storico e un cattivo scrittore, che però è per noi utile per alcuni fatti recenti di cui è testimone diretto, e perché riporta - acriticamente, e questo è un vantaggio, perché lo fa senza cambiare nulla notizie tratte da fonti precedenti che per noi sono altrimenti perdute. ${ }^{10}$

7 Di recente in effetti sulla vicenda è stato scritto un romanzo: F. Prosperi, Due vele per un sogno, Milano, Mursia, 2009.

8 Su di lui cfr. P. Tomea, Per Galvano Fiamma, Italia medioevale e umanistica 39 (1996), 77-120; S. A. Céngarle Parisi, Introduzione, in S. A. Céngarle Parisi - M. David (a cura di), La Cronaca estravagante di Galvano Fiamma, Milano, Casa del Manzoni, 2013, 1-196.

9 P. Chiesa, Galvano Fiamma fra storiografia e letteratura, in Courts and Courtly Cultures in Early Modern Europe. Models and Languages, Roma, Viella, 2016, 77-92, da integrare con Id., «Summa cronicarum». Un'opera incompiuta e perduta di Galvano Fiamma, Filologia mediolatina 24 (2017), 305-21.

10 La affermazioni di consapevolezza storiografica di Galvano sono discusse da 
Questo è appunto il caso della notizia di cui stiamo parlando. La Cronica universalis è un'opera che risponde sotto ogni aspetto al profilo intellettuale che abbiamo fatto dell'autore. ${ }^{11} \grave{E}$ concepita come una storia del mondo dalla Creazione all'età di Galvano, e questo ambizioso piano è presentato nel prologo; ma dei 15 libri previsti ne vengono scritti solo tre e un moncone del quarto: l'opera si conclude all'epoca del re biblico Ioas (IX sec. a.C.), un'interruzione che non pare imputabile a perdita fortuita di materiale, ma a incompletezza originaria. L'opera è forse l'ultima dell'autore in ordine cronologico, ed era quella che sviluppava un progetto più sistematico; ma tale progetto è inficiato da un grande disordine, dovuto anche al fatto che manca ogni rifinitura. È tramandata in un unico manoscritto della fine del Trecento, ${ }^{12}$ che si trovava un tempo presso la biblioteca del monastero milanese di Sant'Ambrogio ed è oggi in una collezione privata negli Stati Uniti $;^{13}$ il testo è inedito, ma la sua pubblicazione è prevista come esito di un progetto didattico attivo presso l'Università degli Studi di Milano (Dipartimento di Studi Letterari Filologici e Linguistici).

Buona parte del terzo libro della Cronica universalis è occupata da una lunghissima digressione geografica, riservata soprattutto a descrivere le terre esotiche: l'Asia anzitutto, di recente riscoperta dalle esperienze

M. Zabbia, La specificità del lavoro di storico secondo Galvano Fiamma, in In presenza dell'autore. L'autorappresentazione come evoluzione della storiografia professionale tra basso Medioevo e Umanesimo, Napoli, University of Napoli Press, 2018, 55-78.

11 Su quest'opera cfr. P Chiesa, «Ystorie Biblie omnium sunt cronicarum fundamenta fortissima». La «Cronica universalis» di Galvano Fiamma (ms. New York, collezione privata), Bullettino dell'Istituto Storico Italiano per il Medioevo 118 (2016), 179-216. Per la posizione dell'opera all'interno della produzione del Fiamma cfr. anche Id., «Summa cronicarum» cit.

12 Il manoscritto è vergato da un copista di nome Pietro Ghioldi (Petrus de Guioldis), che è responsabile della trascrizione di ulteriori codici contenenti altre opere del Fiamma. Su di lui cfr. Céngarle Parisi, Introduzione cit., pp. 90-6; Chiesa, Galvano Fiamma fra storiografia e letteratura cit., 83-87. Gli altri codici copiati dal Ghioldi e contenenti opere fiammesche sono i manoscritti Ambrosiano A 275 Inf, Trivulziano 1438 e Braidense AE X 10. Tutti questi codici hanno la medesima fattura e impaginazione, e rispondono evidentemente a un progetto sistematico di copiatura delle opere di Galvano, effettuata circa mezzo secolo dopo la sua morte. L'attuale manoscritto della Cronica universalis costituiva un tempo la parte finale di quello che oggi è il codice Braidense.

13 Il proprietario ha generosamente concesso a chi scrive di effettuare la riproduzione fotografica dell'intero codice. 
dei viaggiatori, come Giovanni di Pian di Carpine, Marco Polo, Giovanni di Montecorvino; le regioni del nord, fino alla Scandinavia e oltre; le isole atlantiche, compresa quella mitica di san Brendano. L'ultima sezione della digressione geografica è riservata all'Etiopia, che viene descritta lungamente e con una dovizia di particolari ignota alle altre parti della Cronica. ${ }^{14} \mathrm{Si}$ incomincia con la definizione geografica (sub tropico Cancri circa equinoctialem Ethiopia est duplex. Una dicitur orientalis, altera dicitur occidentalis), a detta di Galvano tratta da Ptholomeus. ${ }^{15}$ Si passa poi alla situazione politica e religiosa del paese. L'Etiopia è governata da un potentissimo imperator cristiano, cui obbediscono 74 reges de corona e oltre 300 barones, quasi tutti cristiani. I bambini vengono battezzati, e subito dopo vengono segnati con un marchio di appartenenza tribale, per poterli riconoscere e catturare se fuggono presso un altro signore. L'imperator combatte di continuo contro i musulmani, e sempre li sconfigge; non riconosce l'autorità della Chiesa di Roma, ma soltanto perché non ci sono contatti: gli scambi sono resi difficili da deserti invalicabili e dalla presenza dei musulmani che impediscono il passaggio. L'autorità religiosa è esercitata da un papa christianissimus, chiamato Preytzan, che ha sotto di sé 2667 episcopati; sarebbe pronto a sottomettersi al papa romano, ma non può farlo per le ragioni già dette. Ci sono chiese con tetti d'oro purissimo; il battesimo viene amministrato secondo il rito romano, e in modo simile a Roma, con qualche piccola differenza, è officiata anche la messa; il clero porta vesti diverse a seconda del grado di ordinazione. I santi più venerati sono la Vergine, Giovanni Battista, gli Apostoli, i Padri del Deserto; un culto particolare hanno i primi evangelizzatori di quella terra, l'apostolo Matteo e l'eunuco della regina Candace, di cui parlano gli Atti degli Apostoli (cap. 8). Si raccontano poi un paio di aneddoti ambientati nel paese: una guerra contro i musulmani vinta grazie all'im-

14 Il testo della sezione è trascritto in P. Chiesa, Galvano Fiamma e Giovanni da Carignano. Una nuova fonte sull'ambasceria etiopica a Clemente $V$ e sulla spedizione oceanica dei fratelli Vivaldi, Itineraria 17 (2018), 63-107: 66-69.

15 Cioè probabilmente da uno dei manuali geografici latini di derivazione araba, le notizie dei quali erano ascritte - ed effettivamente in ultima analisi risalivano - ai trattati di Tolomeo. Coordinate sull'Etiopia molto simili a quelle fornite da Galvano si leggono ad esempio nel diffusissimo De sphaera mundi di Giovanni di Sacrobosco (L. Thorndike, The Sphere of Sacrobosco and its Commentators, Chicago, The University of Chicago Press, 1949, 107), che veniva usato come introduzione all'Almagesto. 
piego di babuini addestrati a tirare con l'arco; il diritto che si arrogano gli inviati dell'imperator Ethiopie, quando passano in terra musulmana, di portare una croce eretta davanti al loro passaggio; la focatura in fronte con il segno di una croce, che rivela i pellegrini provenienti dall'Etiopia.

Le notizie che abbiamo qui sintetizzato sono del tutto inedite per l'Occidente medievale. L'idea che universalmente si aveva dell'Ethiopia era quella veicolata dalle fonti enciclopediche tardoantiche, in particolare da Solino, raccolte poi da Isidoro di Siviglia e da lui trasmesse ai secoli successivi. Secondo queste fonti, l'Ethiopia era una terra primitiva e selvaggia, abitata da genti mostruose e belve feroci; una terra aliena e inaccessibile, dove favolose ricchezze si intrecciavano alla più crudele barbarie. Al contrario, Galvano ci fornisce l'immagine di una regione civile, e per di più cristiana; neanche una parola su serpenti o leoni, e nemmeno sulla nigritudo degli abitanti, che tutte le fonti precedenti sottolineavano con forza, spesso per evincerne un'ascendenza demoniaca. Una terra molto simile all'Europa, almeno per appartenenza religiosa e relativi riti.

Da dove arrivano queste notizie? Galvano sembra cosciente dell'eccezionalità di quanto sta raccontando, e distingue la sezione - caso unico in tutta la Cronica universalis - con un titolo specifico, quello di Ystoria Ethiopie. Non si trattiene dal fare propri commenti: a proposito dell'imperator, egli osserva che risulta molto più potente del suo omologo occidentale, che ha sotto di sé soltanto 22 re al posto di 74; e lo stesso può dirsi del papa etiope, che sopravanza quello romano per quasi 2000 vescovi in più. Ma, soprattutto, in due occasioni ci svela la fonte che sta utilizzando, dobbiamo supporre abbastanza fedelmente - se non per il dettato verbale, almeno per il contenuto -, come è nelle sue abitudini: un Tractatus de mappa Ianuensi quam composuit sacerdos Sancti Marchi de Ianua.

Il sacerdos di cui si parla è un personaggio di primissima importanza nella storia della cartografia. Si tratta di Giovanni da Carignano, un prete che officiò la chiesa di San Marco in Molo, all'interno del porto di Genova, nei primi decenni del Trecento, e che è celebre per avere disegnato uno dei primi planisferi di cui si abbia notizia: una mappa dell'Europa e dei mari e delle regioni circonvicine, decorata con stemmi cittadini e nobiliari e corredata da cartigli esplicativi ${ }^{16}$ Che fosse autore di un Tractatus de mappa, dove si parlava fra l'altro dell'Etiopia,

16 Th. Fischer, Sammlung mittelalterlicher Welt- und Seekarten italienischen 
si sapeva da una notizia piuttosto tarda, riferita dal Supplementum cronicarum del bergamasco Giacomo Filippo Foresti (1496); ${ }^{17}$ ma per il resto l'opera sembrava perduta. Il resoconto ben più ampio e circostanziato fornito da Galvano, che è probabilmente la fonte del Foresti, permette ora di capire meglio la natura del Tractatus: uno scritto accessorio a una mappa (il planisfero che oggi conosciamo, o a un'altra carta tracciata da Giovanni), in cui erano depositate notizie aggiuntive che, per la loro estensione, non potevano essere accolte all'interno del disegno.

Galvano attinge dunque, per sua dichiarazione, le notizie sull'Etiopia dal Tractatus di Giovanni da Carignano; e questi a sua volta le ricava da una fonte sorprendente. Galvano racconta che anno domini MCCC l'imperator Ethiopie scrisse una lettera al rex Hispanie; di questa lettera riporta il testo in latino, che contiene una proposta di alleanza militare contro i musulmani, e descrive gli elementi protocollari (tre croci tracciate in rosso all'inizio, altre tre croci analoghe alla fine, un sigillo in oro). La consegna della lettera fu affidata a XXX ambaxiatores; ma quando essi giunsero in Occidente scoprirono che nel frattempo il rex Hispanie era morto, e cambiarono la loro destinazione. Si recarono dapprima ad Avignone ad pappam Clementem; quindi iverunt Romam visitare corpora apostolorum Petri et Pauli; passarono poi per Genova; si diressero ad sanctum Iacobum de Galitia; infine per mare redierunt in regionem suam. Durante la sosta a Genova in scriptis dimiserunt multa de factis sue contrate, e a questo scritto attinge evidentemente Giovanni da Carignano, e per suo tramite Galvano.

Se le informazioni che fornisce il Fiamma sono esatte, veniamo così a conoscere molti dettagli su quello che sembrerebbe essere il primo contatto diplomatico fra Ethiopia e Europa occidentale nel medioevo, quasi un secolo in anticipo rispetto alle attestazioni successive. Gli elementi cronologici forniti - l'anno MCCC, un'indicazione probabilmente incompleta o arrotondata; il pontificato di pappa Clemens, evidentemente Clemente V (1307-1314); la morte di un rex Hispanie,

Ursprungs und aus italienischen Bibliotheken und Archiven, Venezia, Ongania, 1886, 117 126. La mappa è andata distrutta durante la Seconda Guerra Mondiale, ma ne esistono delle riproduzioni fotografiche, anche se di non eccelsa qualità; la migliore è quella conservata all'Archivio di Stato di Firenze, Carte nautiche, geografiche e topografiche 2.

17 Stampato a Venezia, presso Bernardino Benalio (GW M10969; ISTC ij00208000); il passo è ai ff. 17v-18r del libro VIII. 
che dato il periodo può essere solo Ferdinando IV di Castiglia e Léon (1295-1312), che aveva in effetti combattuto contro i musulmani a Gibilterra - inducono a collocare la permanenza in Europa degli ambasciatori negli anni $1312-1315 .{ }^{18}$ La stesura del Tractatus de mappa si data entro il 1330, anno della morte di Giovanni da Carignano; un lasso di tempo piuttosto breve rispetto agli eventi narrati, che costituisce un elemento a favore del fatto che la narrazione sia affidabile.

Ma gli ambasciatori erano davvero etiopi? E quanto di ciò che racconta Galvano corrisponde alla realtà dell'Etiopia di allora? Il termine Ethiopia era usato nel medioevo in un senso assai diverso da quello di oggi, attribuito com'era all'intera Africa subsahariana; inoltre, nella geografia dell'epoca veniva comunemente accettata la contiguità fra Etiopia e India, di ascendenza tolemaica, come se di due parti di una medesima macroregione si trattasse. ${ }^{19}$ Qualcuna delle notizie riportate da Galvano erano già note, in forma molto sintetica, dal Supplementum cronicarum del Foresti, che probabilmente le ricavava da lui; data l'età tardiva e l'isolamento della fonte, questa testimonianza era guardata con sospetto. ${ }^{20}$ Vari studiosi hanno dubitato in particolare che gli ambasciatori provenissero davvero dall'Africa orientale: c'è chi ha supposto si trattasse di indiani, oppure di siriaci, o anche di etiopi veri e propri, ma di stanza a Gerusalemme, dove vi era un monastero della loro Chiesa. ${ }^{21}$ Altri hanno osservato che certi tratti della figura dell'imperator Ethiopie ricordano quelli del Prete Gianni, il fantomatico sovrano

18 Manca qualsiasi riscontro documentario avignonese dell'ambasciata. Un'ipotesi è che gli ambasciatori siano giunti alla meta nella vacanza fra la morte di Clemente V (20 aprile 1314) e l'elezione del papa successivo, Giovanni XXII (7 agosto 1316).

19 Cfr. P. Schneider, L'Éthiopie et l'Inde. Interférences et confusions aux extrémités du monde antique (VIIIe siècle avant J.-C.-VIe siècle après J.-C.), Rome, École française de Rome, 2004 (Collection de l'École française de Rome, 335), pp. 337-340.

20 Così G. F. Beckingham, Ethiope and Europe (1200-1650), in The European outthrust and encounter. The first phase, c. 1400 - c. 1700. Essays in tribute to David Beers Queen on his 85 th birthday, Liverpool, Liverpool University Press, 1994, 77-96: 80-81; A. Knobler, Mythology and diplomacy in the age of exploration, Leiden-Boston, Brill, 2017, 36 n. 20; V. Krebs, Re-examining Foresti's Supplementum Chronicarum and the 'Ethiopian' embassy to Europe of 1306, Bulletin of the School of Oriental and African Studies 82/3 (2019), 493-515.

21 Cfr. fra gli altri Fischer, Sammlung mittelalterlicher Welt- und Seekarten cit., 
dell'India conosciuto per una diffusissima lettera fittizia in cui magnifica la grandezza del suo regno; e che lo stesso nome del papa christianissimus degli Etiopi, Preytzan, sembra una variante grafico-fonetica di quello. Non ci prendiamo perciò la responsabilità di affermare che quel gruppo di forestieri fosse realmente composto da etiopi, nel senso antico o moderno del termine; in ogni caso, tali vennero considerati a Genova.

Impossibile però pensare che la vicenda sia semplice di frutto di fantasia, per la qualità della fonte - Giovanni da Carignano, prete del porto e pioniere della cartografia - e per le informazioni riferite, del tutto diverse dalle credenze vulgate in Occidente a proposito dell'Etiopia. Alcuni elementi concreti non possono che rispecchiare un'esperienza, diretta o mediata: si pensi agli elementi diplomatici della lettera al rex Hispanie, o alla focatio imposta ai bambini come segno di appartenenza tribale, o all'abbigliamento del clero. Quanto al Prete Gianni, non si può dubitare che il testo della sua lettera fittizia sia presente a chi scrive - Giovanni o Galvano -, che ne trae le informazioni sull'organizzazione dell'impero, diviso in una settantina di regni; stupisce al contrario non trovare nella descrizione dell'Etiopia nessuno degli elementi fantastici del meraviglioso regno del Presbyter, non pietre magiche o animali esotici, né spettacolari palazzi o apparati di corte. Se le notizie della Cronica universalis derivassero dall'Epistola si può supporre che la descrizione sarebbe stata ben diversa. Val la pena semmai ricordare che il planisfero di Giovanni da Carignano è una delle prime fonti occidentali a rappresentare un'Etiopia cristiana; ${ }^{22}$ ci si può chiedere perciò se la trasposizione del regno del Presbyter dall'India all'Etiopia, dove esso è abitualmente collocato a partire dalla metà del Trecento, non dipenda proprio da quanto si era venuto a conoscere

p. 119; Revelli, Cristoforo Colombo cit., pp. 252-253; C. F. Beckingham, An Ethiopian embassy to Europe, c. 1310, Journal of Semitic Studies 43 (1998), 337-346; A. Kurt, The search for Prester John, a projected crusade and the eroding prestige of Ethiopian kings, c.1200 - c.1540, Journal of Medieval History 39/3 (2013), 297-320: 308; M. Salvadore, The African Prester John and the birth of Ethiopian-European relations, 1402-1555, London-New York, Routledge, 2017, 2-3; Knobler, Mythology and Diplomacy cit., 36.

22 Nel planisfero l'area africana orientale, posta nell'angolo inferiore destro della mappa e purtroppo assai poco leggibile, sembra costellata da simboli di croci; non vi è, almeno in apparenza, alcun cartiglio che faccia riferimento al Prete Gianni, come sarà abituale nelle carte prodotte a Trecento inoltrato. 
attraverso l'ambasciata. Un grande regno cristiano esisteva davvero: non in Asia, però, ma in Africa.

$$
* * *
$$

\section{E i fratelli Vivaldi? L'ultimo paragrafo dell'Hystoria Ethiopie reci-} ta cosi:

Non solum ex ipsis ad nos pervenerunt supradicti embaxiatores, imo etiam ex nostris aliquando ad ipsos transierunt, prout ipsi XXX embaxiatores in civitate Ianuensi narraverunt. Certum est enim quod anno Domini MCCLXXXX due gallee Ianuensium, in quibus erant plus quam $\mathrm{VI}^{\mathrm{c}}$ viri christiani et aliqui clerici $<\ldots .{ }^{23}$ et ipsarum galearum admirati sunt dicti Ubertus de Savigono et []$^{24} \mathrm{Hii}$ transierunt per strictum Yspanie et navigantes mare Athlanticum pervenerunt ad Ethiopiam que est ultra equinoctialem. Et cum deficerent eis victualia, descenderunt ad terram et cuncta que inveniebant diripiebant, eo quod fame perirent; et capti fuerunt et ducti sunt ad imperatorem Ethyopie supradictum; qui audito quod essent christiani et subditi ecclexie Romane, ipsos libenter vidit et multum ipsos honoravit et posuit eos in magnis offitiis. Et numquam redierunt Ianuam, quia per mare non fuerunt ausi redire propter pericula que nullus homo poset cogitare; nec per terram potuerunt redire quia sarraceni sunt in medio, qui totis viribus prohybent quod christiani non vadant in Ethiopiam nec Ethyopes veniant ad nos, timentes ne ligam facerent contra sarracenos, quia sarraceni omnes essent mortui. Embaxiatores supradicti imperatoris Ethyopie qui fuerunt in Ianua, qui viderunt ipsos Ianuenses in Ethyopia, ista narraverunt.

Non furono soltanto quegli ambasciatori etiopi a venire da noi, ma una volta anche alcuni dei nostri andarono da loro; lo raccontarono quei trenta ambasciatori nella città di Genova. Si sa che nell'anno 1290 due galee genovesi, con imbarcati più di 600 cristiani e alcuni chierici $<\ldots$; ; i comandanti erano Uberto di Savignone e [ ]. Essi oltrepassarono lo stretto di Spagna e, navigando per il mare Atlantico, giunsero nell'Etiopia, a sud dell'Equatore. Non avevano più viveri; scesero a

23 Manca apparentemente almeno il verbo della frase.

24 Nel manoscritto è lasciato in questo punto uno spazio bianco equivalente a una ventina di caratteri; evidentemente si trovava qui in origine il nome di un secondo personaggio, che l'estensore del testo non conosceva o non riusciva a decifrare. 
terra e si misero a razziare tutto ciò che trovavano, perché morivano di fame. Furono catturati e portati dall'imperatore che si è detto, e questi, saputo che erano cristiani e sudditi della Chiesa romana, li incontrò volentieri, li onorò molto e conferì loro grandi dignità. Non rientrarono più a Genova: non se la sentirono di tornare per mare, per i pericoli che vanno oltre ogni immaginazione, e non poterono tornare per terra, perché in mezzo stanno i musulmani, che cercano di impedire con tutte le loro forze che i cristiani vadano in Etiopia e che gli Etiopi vengano da noi, perché temono che si alleino contro di loro, cosa che sarebbe la loro fine. Tutto questo lo narrarono gli ambasciatori dell'imperatore di Etiopia che furono a Genova, e che videro quei Genovesi in Etiopia.

La spedizione di cui parla Galvano - ma certo già Giovanni da Carignano, perché soltanto da una fonte genovese il Fiamma poteva ricavare informazioni del genere - non può essere che quella di cui parla anche Iacopo Doria. Vi sono alcune piccole, anche se significative, differenze nei dettagli - l'anno è il 1290, e non il 1291; i marinai sono l'esagerato numero di 600; i religiosi sono in numero indeterminato; il comandante non è un Vivaldi, ma Uberto di Savignone (un castello non lontano da Genova); non si fa menzione di Tedisio d'Oria ${ }^{25}$-, ma indubbiamente l'evento narrato è il medesimo. La maggiore novità sta però in quanto Galvano dice della sorte dei naviganti: essi non scomparvero fra i flutti, ma approdarono in Etiopia, dove vennero onorevolmente accolti, sia pure dopo qualche turbolenza.

La notizia data da Giovanni sull'esito della spedizione toglie dal loro isolamento alcune voci più tarde, considerate romanzesche, che ugualmente parlano nebulosamente della sopravvivenza dei marinai in Africa. Il Libro del conosçimiento de todos los reynos, una sorta di romanzo di viaggio catalano scritto verso la fine del Trecento, ricorda la spedizione di un genovese di nome Sorleone, che vanamente si avventura nella regione di Magdasor (si è proposta l'identificazione con Mogadiscio) alla ricerca del padre, che faceva parte della spedizione oceanica:

Et dixeron me en esta çibdad de Grançiona que fueron y traidos los ginoveses que escaparon de la galea que se quebró en Amenuan, et de la otra galea que escapó nunca sopieron qué se fizo... An esta çibdad de Magdasor me dixeron de un ginoves que dezian Sorleonis que fuera y en

25 Su queste differenze cfr. Chiesa, Galvano Fiamma e Giovanni da Carignano cit., 103. 
busca de su padre que fuera en una de las galeas de que ya conté de suso. Et fizieronle toda onrra. Et este Sorleonis quysiera traspasar al inperio de Graçiona a buscar a su padre, et este Enperador de Magdasor non le consintio yr por que la yda era dubdosa por que el camino es peligroso. ${ }^{26}$

A quanto mi dissero nella città di Graçiona, lì erano stati portati i genovesi che erano scampati al naufragio della loro galea ad Amenuan; dell'altra galea, che si era salvata, non conoscevano la sorte... Nell'altra città di Magdasor mi raccontarono di un genovese, di nome - dicevano - Sorleone, che era andato lì in cerca di suo padre, che era partito con le galee di cui si è parlato. Gli avevano tributato tutti gli onori; Sorleone voleva passare nell'impero di Graçiona per cercarlo, ma l'imperatore di Magdasor glielo impedì perché il viaggio era troppo incerto, per i pericoli lungo la via.

Nella seconda metà del Quattrocento il cosiddetto Itinerario di Antoniotto Usodimare - una raccolta poco organica di notizie geografiche, in parte ricavate da mappe - torna a citare la spedizione dei Vivaldi, e anche in questo caso si parla della sopravvivenza dell'equipaggio in Africa:

Anno Domini $\mathrm{M}^{\circ} \mathrm{CCLXXXX}$ recesserunt de civitate Ianue due galee patronizate per dominum Vadinum et Guidum de Vivaldis fratres, volentes ire in levante ad partes Indiarum; que gallee multum navigaverunt. Sed quando fuerunt dicte due gallee in hoc mari de Ethiopia, ${ }^{27}$ una earum se reperit in fundo sicco, per modum quod non poterat ire nec ante navigare. Alia vero navigavit et transivit per istud mare usque dum venirent ad civitatem unam Ethiopie nomine Menam, capti fuerunt et detempti ab illis de dicta civitate qui sunt christiani de Ethiopia, submissis presbitero Iohanni. Civitas ipsa est ad marinam prope flumen Sion. Predicti fuerunt taliter detempti quod nemo illorum a partibus illis unquam redidit. Quae predicta narraverunt mercatores Ethiopani in civitate Cari. ${ }^{28}$

26 El libro del conoscimiento de todos los reinos (The Book of knowledge of all kingdoms), ed. N. F. Marino, Tempe, Arizona Center for Medieval and Renaissance Studies, 1999, 60-64. Cfr. Magnaghi, Precursori di Colombo? cit., 51-82.

27 Nel codice che riporta il testo (Genova, Biblioteca Universitaria, B.I.36, ff. 8v-9r) la parola Ethiopia è stata successivamente corretta in Ghinoia (la 'Guinea'), evidentemente per spostare in una regione più verosimile, la più raggiungibile Africa Occidentale, l'intera vicenda.

28 Cfr. Surdich, L'impresa dei fratelli Vivaldi cit., 67. 
Nell'anno 1290 partirono da Genova due galee, armate dai fratelli Vadino e Guido de' Vivaldi, che volevano andare in Oriente, nelle regioni dell'India. Le galee fecero una lunga navigazione; ma quando arrivarono nel mare dell'Etiopia, una delle due si incagliò in una secca, e non riuscì più a proseguire. L'altra continuò il viaggio e attraversò quel mare fino a giungere a una città dell'Etiopia chiamata Mena. Lì furono catturati e tenuti prigionieri dagli abitanti, che sono cristiani dell'Etiopia, sottomessi al Prete Gianni. Quella città si trova sul mare, presso il fiume Sion. La loro prigionia fu tale che nessuno di loro poté poi ritornare da lì. Tutto questo fu raccontato da mercanti etiopi nella città del Cairo.

E probabile che queste tarde notizie risalgano, in via diretta o indiretta (e con tutte le trasformazioni indotte dal tempo e dall'oralità), al Tractatus de mappa di Giovanni da Carignano, e dunque alla presunta ambasciata etiopica; la testimonianza di Galvano ci permette ora di recuperare il punto di partenza.

Arrivarono davvero in Etiopia i 'fratelli Vivaldi' (o Uberto di Savignone, o chiunque altro si trovasse a bordo di quelle galee)? Quasi sicuramente no. La circumnavigazione dell'Africa richiedeva competenze e mezzi tecnici che alla fine del Duecento erano impensabili; soltanto due secoli più tardi, dopo vari tentativi parziali, poté essere realizzata da Vasco da Gama. Questa storia è dunque, con ogni probabilità, falsa; ma era quella che a Genova volevano sentirsi raccontare. Forse gli ambasciatori 'etiopici' - ammesso che tali fossero - avevano parlato di altri occidentali che avevano sì raggiunto la loro terra, come all'inizio del Trecento ormai accadeva, ma per la più semplice via del Mar Rosso, e Giovanni da Carignano fraintese; forse non avevano parlato affatto di quegli uomini, ma il prete del porto costruì per i nipoti degli scomparsi un messaggio di consolazione, e per la marineria di Genova un mito fondativo, inventando un esito fausto per una spedizione fallita. 